\title{
Propagation of $A \beta$, tau and $\alpha$-synuclein pathology between experimental models and human reality: prions, propagons and propaganda
}

\author{
Toshiki Uchihara ${ }^{1} \cdot$ Benoit I. Giasson $^{2} \cdot$ Werner Paulus $^{3}$
}

Published online: 29 December 2015

(C) Springer-Verlag Berlin Heidelberg 2015

In 2008, Acta Neuropathologica published a review cluster on genetically engineered animal models of neurodegenerative diseases comparing their similarities and differences to human neuropathology [8]. Although these models (and the ones generated since) recapitulate some pathological features of the human diseases, their overall pathology did not closely resemble what is typically observed in human brains. Nevertheless, these models were considered more or less useful as tools to try to understand mechanisms of specific proteinopathies. Although it remains to be clarified how the distribution of each cytopathology is regulated, it is possible to modify their distribution using specific promoters so that pathological proteins are artificially driven to selected areas or cell types in the nervous system. Importantly, it still remained to be explained how the distributions of protein inclusions are dictated by disease states in human brains. In recent years, some of these experimental modeling approaches have been expanded to exacerbate the

Toshiki Uchihara

uchihara-ts@igakuken.or.jp

$\triangle$ Benoit I. Giasson

bgiasson@ufl.edu

Werner Paulus

werner.paulus@uni-muenster.de

1 Laboratory of Structural Neuropathology, Tokyo Metropolitan Institute of Medical Science, 156-8506, 2-1-6 Kamikitazawa, Setagaya-ku, Tokyo 156-8506, Japan

2 Department of Neuroscience, Center for Translational Research in Neurodegenerative Disease, McKnight Brain Institute, University of Florida, 1275 Center Drive, PO Box 100159, Gainesville, FL 32610-0159, USA

3 Institute of Neuropathology, University Hospital Münster, Pottkamp 2, 48149 Münster, Germany localized inducing of lesions by cerebral or peripheral injections of preparations containing synthetic or biologically derived aggregate-inducing amyloid protein "seeds", resulting in subsequent spread of pathological inclusions. These studies were based on the assumptions that (1) endogenous proteins can be modified to be pathological as they are endowed with a property to template themselves and polymerize to form pathological inclusions, and (2) this molecular process can progressively spread along neuronal processes and synapses, which is supposed to represent the reality of human brain. While these two different aspects are sometimes considered "prion-like", this review cluster addresses whether such experimental approaches represent similarities, which could be grouped under a catch-all umbrella "prion" or "prion-like" and whether there is sufficient evidence from the human neuropathology counterpart to support such a unified mechanism. To avoid experimental or human bias, researcher pairs of a experimental neuroscientist and a human neuropathologist were selected to provide a balanced view of the current state of experimental versus descriptive findings in terms of explaining the distribution/spread of pathological lesions for some of the major proteins ( $\alpha$-synuclein, tau and $A \beta$ ) often found as inclusions in the brains of patients with neurodegenerative diseases. This binocular view hopefully provides a stereoscopic and more rigorous interpretation, but also highlights important discrepancies between the two aspects. As expected, this challenge introduced conflicting views in each pair, but we feel that in the end all participants were successful in demonstrating both similarities and differences between propagation experiments and human neuropathology together with their shortcomings as summarized below. 


\section{Similarities and differences in pathological and experimental studies}

Experimental studies using $\alpha$-synuclein transgenic mice, but in some cases even using wild type mice, have demonstrated that brain inoculations with pre-aggregated $\alpha$-synuclein "seeds", capable of initiating protein templating of endogenous $\alpha$-synuclein to form aggregates, can result in neuronal spread of $\alpha$-synuclein inclusion pathology in either retrograde or anterograde direction and even sometimes transsynaptic spread [7]. This is in contrast with a predominantly retrograde intraneuronal spread of $\alpha$-synuclein pathology in human brain, which occurs preferentially in neurons with hyperbranching axons, suggesting that it is probably not only molecular propensities of $\alpha$-synuclein itself but also underlying neuroanatomical structure that influences templating of $\alpha$-synuclein-related neurodegeneration. It is proposed to call such structural constraint "structural template", which facilitates intraneuronal spread of $\alpha$-synuclein pathology predominantly in the retrograde direction. On the other hand, $\alpha$-synuclein lesion distribution is so variable that it is hardly explained by simple neuroanatomical and transsynaptic spread in serial chain along a predefined sequence as assumed by most experimental studies and Braak's staging scheme. To explain the variable distribution of $\alpha$-synuclein lesions in the human nervous system, a novel concept of "multifocal Lewy body disease" (emphasized here in the $\alpha$-synuclein review) is proposed [7]. Independent and parallel appearance of focal Lewy body pathology in multiple neuronal groups, regardless of their transsynaptic connections, may better explain the variable clinicopathological pictures, more extensively recognized recently in a clinical setting. In fact, this concept is consistent with spreading patterns observed in some experimental animal studies, where strict transsynaptic spread was not observed and also involving transmission via glial cells. Furthermore, experimental evidence indicates that non-conformational templating mechanisms, including neuroinflammation, neurotoxicity/injury and general perturbation of proteostasis, can also play some roles in the progressive spread of $\alpha$-synuclein inclusion pathology. This notion was recently further demonstrated by the finding that the simple brain injection of a spinal cord homogenate from a normal mouse without any aggregated $\alpha$-synuclein can induce the formation of widespread CNS $\alpha$-synuclein inclusion pathology in an $\alpha$-synuclein transgenic mouse model, similar to what had been previously inferred in prior studies as a "prionlike" mechanism [5]. Therefore, at least in animal models, multiple mechanisms can induce the spread of $\alpha$-synuclein pathology. It could be mediated even by something other than $\alpha$-synuclein, suggesting that some intrinsic cascades can trigger the formation of $\alpha$-synuclein inclusion pathology, but it remains to be determined if all these findings can be translated to observations in human brains.
In contrast with $\alpha$-synuclein, tau pathology in Alzheimer's disease (AD) predominantly spreads in the anterograde direction as seen in the dentate gyrus, even if experimental studies demonstrated the possibility of either anterograde or retrograde spread $[2,4]$. As pointed out by Lewis and Dickson in this issue [4], it is not yet clear whether such anterograde spread is shared with other tau-related diseases such as progressive supranuclear palsy, corticobasal degeneration, or Pick's disease. Moreover, most propagation studies used "tau" without discriminating the effects of specific isoform(s) or combinations of isoforms, which could be an important confound since there exist clear differences in the biochemical and pathological signatures of tau in different tauopathies [3]. In addition, the role of neuronal function and electrical activity along specific neuroanatomical pathways has been associated with the patterns of spread of tau-positive lesions in $\mathrm{AD}$, summarized as "What wires together, fires together and degenerates together." [6], which may indicate "functional template". Because dynamic polarization of each neuron is serially chained to form functional networks to facilitate unidirectional conduction of electrical activities [1], it is likely that the anterograde spread of tau pathology and possibly tau aggregates traces this functional conduit. In contrast, the predominant retrograde intraaxonal spread of $\alpha$-synuclein pathology and possibly $\alpha$-synuclein aggregates hardly follows such anterograde conduction. In any case, how such functionality relates to molecular templating is still largely unclear.

The presentation of $\mathrm{A} \beta$ deposition in human brains is more disordered and less stratified probably because of its extracellular nature and diversity of $A \beta$ deposits, although patterns and distribution gradients exist. Even though hierarchical distribution as formulated by Thal and CERAD staging are current frameworks for $A \beta$ spread [2], their underlying mechanisms are poorly explained. As described in the review by Eisele and Duyckaerts in this issue [2], experimental studies have provided compelling evidence that brain inoculations of "seeds" of aggregated $A \beta$ can induce the further aggregation of endogenous $A \beta$, which is mechanistically much more straightforward given the extracellular nature of $\mathrm{A} \beta$ peptides. Nevertheless, these studies have not yet provided a clear picture of the predominant mechanism involved in the more restricted spread of $A \beta$ pathology that may include intraneuronal, extraneuronal, and perivascular routes. Furthermore, possible relations between tau and $A \beta$ are still poorly understood, which further complicates the interpretation of the presentation of both types of pathologies in AD. In addition, the effects of the variable compositions of brain $\mathrm{A} \beta$ peptides (i.e. $\mathrm{A} \beta 1-$ $40, A \beta 1-42$ and the numerous other truncated or modified $\mathrm{A} \beta$ species) on the progressive induction and spread of $\mathrm{A} \beta$ aggregation are still poorly understood. Even the toxic mechanisms associated with aggregated $A \beta$ and $A \beta$ oligomers are still a highly contentious issue. 


\section{“Prion-like” versus “propagon" terminology}

Considering the diversities in brain pathology and mechanistic unknowns, the catch-all umbrella term "prionlike" remains poorly defined or diversely defined by different investigators, which may potentially provide the impression that neuropathological cascades involving $\alpha$-synuclein, tau and $A \beta$ are mostly shared, while in reality they are quite different at least in human brains. This impression could be exaggerated to the extent that potential infectivity of these pathological proteins across individuals is overemphasized, which attracted public attention to "propagation", potentially serving as a propaganda in favor of the protagonist researchers. To avoid such confusion, Eisele and Duyckaerts used and expanded the term "propagon" just to describe "what is being propagated or which specific aspect has been investigated" [2]. For example, this terminology can be coupled with specific descriptive terms such as "molecular propagon" or "tissue propagon". Authentic prion protein has the capability to be templated into a pathogenic and infectious state that may spread between organs and organisms regardless of the pathways and mechanisms involved, while in comparison there are clearly biological barriers that thwart this from happening for $\mathrm{A} \beta, \alpha$-synuclein and tau. The mechanistic and biological distinctions that occur at the different spatial levels of the spread of protein inclusions can be more accurately described by applying the "propagon" terminology, allowing more detailed differentiation of each step (molecular level, within tissue, across tissue and from organism to organism), which is highly variable according to the innate properties of each molecule and its relation to the biological/pathological circumstances as well. Using the "propagon" concept, there is no need any more to discuss whether the event in question is "prion-like" or not. On the contrary, the infectious potential of authentic prion protein might have obscured these details. In this sense, the genuine prion protein is just one extreme of propagons. In some ways, the extremist use of "prion-like" terminology is similar to the highly artificial experimental paradigms that have been used in some studies to support the notion of induction and spread of specific proteinopathies. When trying to compare the human pathological findings to experimental studies, it is important to emphasize that some of the latter findings were observed in animal experiments where dosage of inoculation ( $\alpha$-synuclein, tau, $A \beta$ ) in some cases were used at particularly high concentrations, susceptibility of the recipient animals were enhanced by artificial (over) expression of wild type or mutant proteins and tissue disruption at the site of injection might have exacerbated the uptake and spread of aggregated proteins. In human propagon, the pathological protein is likely more tightly subject to structural and functional templates, which may exhibit unidirectional spread along neurites, while this could have been artificially altered in the animal models. Human and experimental propagons likely share common biological mechanisms. However, for providing insights, neither is superior over the other-their analyses are complementary. Experimental studies are often more simple and straightforward to interpret, however, references to the disease states in human brains are necessary to validate that these models reflect "human disease-relevant" progression and propagation, as illustrated in this cluster of review articles. Furthermore, comparison of different molecules demonstrated a contrast in human brain: parallel multifocal occurrence of $\alpha$-synuclein lesions versus serial spread of tau lesions across neurons, which is hardly explained by a catch-all umbrella "prion-like". Instead, human disease-specific details may be uncovered by introducing the more flexible concept of "propagon" so that the disease-specific diagnosis and therapies are tailored. Acta Neuropathologica is pleased to launch this propaganda, hoping to improve our understanding on neurodegenerative diseases so that sufferings of patients from these so-far intractable disorders may be alleviated in the future.

\section{References}

1. Cajal RS (2002) Structure et connexions des neurones. Rev Exp Patol 35(4):433-452. http://www.nobelprize.org/nobel_prizes/ medicine/laureates/1906/cajal-lecture.pdf

2. Eisele Y, Duyckaerts C (2016) Propagation of Abeta pathology: hypotheses, discoveries, and yet unresolved questions from experimental and human brain studies. Acta Neuropathol. doi:10.1007/s00401-015-1516-y

3. Hara M, Hirokawa K, Kamei S, Uchihara T (2013) Isoform transition from four-repeat to three-repeat tau underlies dendrosomatic and regional progression of neurofibrillary pathology. Acta Neuropathol 125(4):565-579. doi:10.1007/s00401-013-1097-6

4. Lewis J, Dickson D (2016) Propagation of tau pathology: hypotheses, discoveries, and yet unresolved questions from experimental and human brain studies. Acta Neuropathol. doi:10.1007/s00401-015-1485-1

5. Sacino AN, Ayers JI, Brooks MM, Chakrabarty P, Hudson VJ 3rd, Howard JK, Golde TE, Giasson BI, Borchelt DR (2016) Non-prion-type transmission in A53T alpha-synuclein transgenic mice: a normal component of spinal homogenates from naive non-transgenic mice induces robust alpha-synuclein pathology. Acta Neuropathol. doi:10.1007/s00401-015-1505-1

6. Seeley WW, Crawford RK, Zhou J, Miller BL, Greicius MD (2009) Neurodegenerative diseases target large-scale human brain networks. Neuron 62(1):42-52. doi:10.1016/j. neuron.2009.03.024

7. Uchihara T, Giasson B (2016) Propagation of alpha-synuclein pathology: hypotheses, discoveries, and yet unresolved questions from experimental and human brain studies. Acta Neuropathol. doi:10.1007/s00401-015-1485-1

8. Uchihara T, Paulus W (2008) Research into neurodegenerative disease: an entangled web of mice and men. Acta Neuropathol 115(1):1-4 